\title{
EXTRACORPOREAL SHOCK WAVE LITHOTRIPSY IN THE TREATMENT OF RENAL PELVICALYCEAL STONES IN MORBIDLY OBESE PATIENTS
}

\author{
V. A. MEZENTSEV \\ Department of Urology, Moscow Regional Scientific Research Clinical Institute, Moscow, Russia
}

\begin{abstract}
Introduction: Management of urolithiasis in morbidly obese patients is usually associated with higher morbidity and mortality compared to non-obese patients. In morbidly obese patients, since the kidney and stone are at a considerable distance from the skin (compared to non-obese patients) difficulty may be found in positioning the patient so that the stone is situated at the focal point of the lithotripter.

Objective: To evaluate the outcomes and cost-efficiency of extracorporeal shock wave lithotripsy (ESWL) in the treatment of renal pelvicalyceal stones sized between 6 and $20 \mathrm{~mm}$ in morbidly obese patients.

Materials and Methods: Using various aids, such as mobile overtable module, extended shock pathway and abdominal compression 37 patients with body mass index more than $40 \mathrm{~kg} / \mathrm{m} 2$ were treated using the Siemens Lithostar-plus third generation lithotripter. The size of renal pelvicalyceal stones was between 6 and $20 \mathrm{~mm}$. Treatment costs for shock wave lithotripsy were calculated.

Results: The overall stone free rate at 3 months of $73 \%$ was achieved. The mean number of treatments per patient was 2.1 . The post-lithotripsy secondary procedures rate was $5.4 \%$. No complications, such as subcapsular haematoma or acute pyelonephritis were recorded. The most effective (87\% success rate) and cost-efficient treatment was in the patients with pelvic stones. The treatment of the patients with low caliceal stones was effective in $60 \%$ only. The cost of the treatment of the patients with low calyceal stones was in 1.8 times higher than in the patients with pelvic stones.

Conclusion: We conclude that ESWL with the Siemens Lithostar-plus is the most effective and cost-efficient in morbidly obese patients with pelvic stones sized between 6 and $20 \mathrm{~mm}$. 87\% success rate was achieved. The increased distance from the skin surface to the stone in those patients does not decrease the success rate provided the stone is positioned in the focal point or within $3 \mathrm{~cm}$ of it on the extended shock pathway. ESWL should not be considered as the first line of treatment in the morbidly obese patients with low caliceal stones where the stone was positioned more than $1 \mathrm{~cm}$ from the focal point on the extended shock pathway.
\end{abstract}

Key words: high-energy shock waves; lithotripsy; kidney calculi; obesity, morbid Int Braz J Urol. 2005; 31: 105-10

\section{INTRODUCTION}

An improvement in the social and economic conditions in the most European countries over the last few decades and the adoption of a more sedentary lifestyle have resulted in an accompanying increase in the number of obese individuals (1). The prevalence of stone disease is also increasing and the combination of these two factors means that more obese and morbidly obese patients are presenting to urologists for the treatment of renal stones (2).

The goal of kidney stone surgical treatment is to achieve maximal stone clearance with minimal morbidity to the patient. Multiple options are currently 
available including ESWL (3), percutaneous nephrolithotomy (PCNL) (2), retrograde intrarenal surgery (RIRS) (4), and in rare cases, open or laparoscopic stone surgery. ESWL has revolutionized the treatment of kidney stone disease and the majority of "simple" renal calculi (about 80 - 85\%) can be treated satisfactorily with ESWL (5).

However, several unique issues arise in the surgical arena with morbidly obese patients: these patients may exceed the weight limits of standard surgical tables and may have skin-to-organ distances that exceed the focal length of the lithotripter or length of standard endoscopic equipment.

The purpose of the study was to evaluate the outcomes and cost-efficiency of ESWL in the treatment of renal pelvicalyceal stones sized between 6 and $20 \mathrm{~mm}$ in morbidly obese patients.

\section{MATERIALS AND METHODS}

We reviewed the clinical records of all patients with morbid obesity that were treated with the Siemens Lithostar Plus, third generation lithotripter by one urologist at the Moscow Regional Scientific Research Clinical Institute between 1995 and 2002. According to the World Health Organization classification patients with body mass index (BMI) more than $40 \mathrm{~kg} / \mathrm{m} 2$ were classified as morbidly obese. During the study period we identified 37 cases, aged $18-72$ years (mean age 45 years). Twenty were male and 17 were female. Patient weights ranged from 123 to 179 $\mathrm{kg}$ (mean $143 \mathrm{~kg}$ ).

Renal colic was the most frequent symptom in 24 patients $(64.9 \%) .12$ patients $(32.4 \%)$ complained of vague abdominal pain. 14 patients (37.8\%) presented with hematuria and 4 patients $(10.8 \%)$ presented with slight fever. Urinalysis and urine culture were performed in 36 patients (97.3\%). $34(91.9 \%)$ patients had intravenous urography (IVU) and all 37 had ultrasonography.

Two patients had renal surgery 4 and 7 years before ESWL. 3 patients were treated by PCNL previously and ESWL was used for treatment of residual fragments. All these 3 patients had nephrostomy at the time of ESWL.
None of our patients had bilateral stones. Pelvic stones were found in 15 patients and 22 patients had caliceal stones, including 11 with low caliceal stones. The stone size was between 6 and 20 $\mathrm{mm}$ ( mean $16 \mathrm{~mm}$ ).

Coexisting medical conditions included hypertension, chronic obstructive airway disease/ asthma, diabetes mellitus and previous deep venous thrombosis.

Criteria for ESWL were symptomatic stone size between $6 \mathrm{~mm}$ and $20 \mathrm{~mm}$ without evidence of active urinary tract infection (UTI). Good renal function was essential.

All the patients underwent ESWL with the Siemens Lithostar Plus, third generation lithotripter. All patients were treated while at the side position using an overtable module. The table was lowered (the z-axis) to its limit and was covered with the protected plastic cover to ensure that the table was secure. The level of energy was between 5 and 7 (mean $6)$. The number of shock waves delivered per session were between 2800 and 3500 (mean 3300). Stones were localized by ultrasound. Seventy-eight sessions of ESWL were performed. The mean number of treatments per patient was 2.1 .

The mobility of the overtable module helped in positioning the stone in the focal point or within 3 $\mathrm{cm}$ of it on the extended shock pathway. In 24 (64.9\%) patients we used an abdominal compression strap to facilitate positioning of the stone within the extended shock pathway. Using the overtable ultrasound module is operator dependant. In our patients it took between 3 and 15 min to locate the stones on the extended shock pathway.

ESWL was performed without analgesics in 11 patients. Fifteen required non-steroidal anti-inflammatory drugs (NSAID) and 11 had NSAID with fentanyl. The interval between treatment sessions was at least 5 days to allow the passage of fragmented debris before the next session.

Average treatment costs for shock wave lithotripsy were calculated as the cost of equipment and its maintenance divided on the number of the treatment sessions performed since the equipment was bought. Nowadays in Russia the salary of medical staff and engineers involved and cost of medication 
used are much lower than in Western countries and hardly contribute in overall treatment expenses. The Siemens Lithostar Plus was bought in 1990. For 12 years 8244 sessions have been done. The costs of the Siemens Lithostar Plus and its maintenance was 2,700.000 DM. Taking into account the aforementioned figures, the average treatment cost for the shock wave lithotripsy session was 327.5 DM or US\$218.3.

\section{RESULTS}

All patients were treated on an outpatient basis, and $2(5.4 \%)$ required hospitalization for postESWL pain. Treatment time was between 35 and 57 min. (mean $45 \mathrm{~min}$ ).

The immediate follow-up after ESWL was by ultrasonography in all patients, followed by a plain abdominal film for patients who had radio-opaque calculi, from which the size of the residual fragments was determined. The treatment was considered successful at 3 months of follow-up if there was no radiological evidence of stones on both ultrasonography and the plain film.

Patients were stratified into 3 groups according to the position of the stone in relation with the focus of shock wave, namely, within $1 \mathrm{~cm}$, between 1 and $2 \mathrm{~cm}$ and between $2-3 \mathrm{~cm}$. All 37 patients were available for follow-up for 3 months.

Table- 1 shows the outcome of ESWL in the treatment of pelvicalyceal stones according to the stone location and the position of the stone in relation to the focus of the shock wave. Tables- 2 and 3 respectively show the summary of the outcome and cost of ESWL according to the position of the stone in relation to the focus of the shock waves and location of the stone in the pelvicalyceal system.

The treatment was considered successful in 27 patients $(73 \%)$. The success rate was lower in

Table 1 - The outcome of ESWL in the treatment of renal pelvicalyceal stones sized between 6 and 20 mm in morbidly obese patients.

\begin{tabular}{lccc}
\hline & Blast Path $<\mathbf{~ c m}$ & Blast Path 1 - 2 cm & Blast Path 2 - 3 cm \\
\hline Pelvic stones & 3 & 7 & 5 \\
Number of ESWL & 3 & 10 & 11 \\
Success rates & $3(100 \%)$ & $6(85.7 \%)$ & $4(80 \%)$ \\
Low caliceal stones & 2 & 4 & 4 \\
Number of ESWL & 7 & 10 & 12 \\
Success rates & $2(100 \%)$ & $2(50.0 \%)$ & $2(50 \%)$ \\
Upper and middle caliceal stones & 4 & 3 & 5 \\
Number of ESWL & 6 & 8 & 11 \\
Success rates & $3(75 \%)$ & $2(75.0 \%)$ & $3(60 \%)$ \\
\hline
\end{tabular}

Table 2 - The summary of the outcome and cost of ESWL in the treatment of pelvicalyceal stones sized between 6 - $20 \mathrm{~mm}$ in morbidly obese patients according to the position of the stone in relation to the focus of the shock waves (blast path).

\begin{tabular}{lccc}
\hline & Blast Path $<\mathbf{1 ~ c m}$ & Blast Path $\mathbf{~} \mathbf{~ 2 ~} \mathbf{~ c m}$ & Blast Path 2 - 3 cm \\
\hline Number of stones & 9 & & 14 \\
Number of ESWL & 16 & 28 & 34 \\
Number of ESWL per stone & 1.8 & 2 & 2.4 \\
Success rates & $8(89 \%)$ & $10(71.4 \%)$ & $9(64.3 \%)$ \\
Cost of treatment (average) & US\$ 393 & US\$ 437 & US\$ 524 \\
\hline
\end{tabular}


Table 3 - The outcome and cost of ESWL in the treatment of pelvicalyceal stones sized between 6 and 20 mm in morbidly obese patients according to the position of the stone in pelvicalyceal system (summary).

\begin{tabular}{lccc}
\hline & Pelvic Stones & Low Caliceal Stones & Upper and Middle Caliceal Stones \\
\hline Number of stones & 15 & 10 & 12 \\
Number of ESWL & 24 & 29 & 25 \\
Number of ESWL per stone & 1.6 & 2.9 & 2.1 \\
Success rates & $13(87 \%)$ & $6 \quad(60 \%)$ & $8 \quad(67 \%)$ \\
Cost of treatment (average) & US\$ 349 & US\$ 633 & US $\$ 458$ \\
\hline
\end{tabular}

the patients treated with the blast path between 2 and $3 \mathrm{~cm}$ than in the patients treated with the blast path less than $1 \mathrm{~cm}, 64.3 \%$ and $89 \%$ respectively $(\mathrm{p}<0.01)$.

The most effective ( $87 \%$ success rate) and cost-efficient treatment was in the patients with pelvic stones. The treatment of the patients with low caliceal stones was effective in $60 \%$ only. The lowest success rate of the treatment $(50 \%)$ was in the patients with low caliceal stones where the stone was positioned more than $1 \mathrm{~cm}$ from the focal point on the extended shock pathway. The cost of the treatment of the patients with low calyceal stones was in 1.8 times higher than in the patients with pelvic stones, US\$ 643 and US\$ 349 respectively.

Two patients $(5.4 \%)$ underwent cystoscopic stent placement for obstruction 3 and 5 days after ESWL. No complications, such as acute pyelonephritis, perirenal or subcapsular haematoma were recorded.

\section{COMMENTS}

Obesity and morbid obesity confer an increased risk of morbidity because of their association with diabetes, cardiovascular and respiratory disease. These impairments will tend to increase during and after surgery, and place the patient at risk of myocardial ischemia and hypoxemia. Surgery in the morbidly obese is more likely to be complicated by thrombo-embolism, wound infection and dehiscence, and respiratory problems (6). Anesthesia and surgery may present a considerable risk for obese patients and should not be undertaken without a full understanding of the potential problems.
Morbidly obese patients carry a physical and psychological burden (7). Surgeons will often send obese patients away to lose weight before they will consider an operation. This is a blow to the obese patient's self-esteem and serves to further erode the relationship between patient and surgeon. In morbidly obese patients attempts at dieting and weight reduction are largely unsuccessful without inpatient treatment, and merely serve to compound their problems by delaying treatment for symptomatic stone disease.

Morbidly obese patients with stones may not present with classic frank pain, but may present with vague complains due to ill-defined body landmarks. 12 patients in our study presented with vague abdominal pain. The response to anti-inflammatory drugs for the relief of renal colic is similar in obese and nonobese patients.

Morbid obesity poses a problem to the successful treatment of kidney stones. The most significant problems in using PCNL for treatment kidney stones in obese patients is the increased risk of nephrostomy tube lose or its displacement (8). The ESWL table may not be able to support the weight of the patient and the increased distance from the skin surface to the stone may render positioning of the stone at the focus of the shock wave impossible (Table-4).

Utilization of the "blast path" (9) is necessary to overcome this problem. The distribution of pressure and the size of focal area of a lithotripter are essential for implementing "blast path" technique (10). The focal area is usually an ellipse. The Table-5 represents the size of focal area for different lithotripters (11). 
Table 4 - Lithotripter specification.

\begin{tabular}{lcc}
\hline Manufacturer & Model & Focal Length $(\mathbf{m m})$ \\
\hline Dornier & DoLiS & 150 \\
Healthronics & Lithotron & 150 \\
Medstone & STS & 153 \\
Siemens & Lithostar & 155 \\
Karl Storz & Modulith & 165 \\
\hline
\end{tabular}

Table 5 - Lithotripter focal areas.

\begin{tabular}{lcc}
\hline Manufacturer & Model & $\begin{array}{c}\text { Size of Focal } \\
\text { Area (mm) }\end{array}$ \\
\hline Edap & Praktis & $27 \times 5$ \\
Storz & Modulith & $28 \times 6$ \\
Dornier & Doli S & $45 \times 5$ \\
Siemens & Lithostar & $80 \times 5$ \\
Healthronics & Lithotron & $38 \times 8$ \\
Medispec & Econolith & $60 \times 13$ \\
Medstone & Medstone & $50 \times 15$ \\
\hline
\end{tabular}

As we can see from the table- 5 the maximum length of focal area of the Siemens Lithostar-plus is $80 \mathrm{~mm}$. This enables an operator to use "blast path" technique when a stone is located a few $\mathrm{cm}$ from the center of focal area. The mobility of the overtable module helped in positioning the stone in the focal point or within $3 \mathrm{~cm}$ of it on the extended shock pathway. We found using the overtable module more flexible in positioning the stone compared to the fluoroscopic module. An operator enables to use different angles between the module and skin to localize the stone at the nearest to the focal area point. In the fluoroscopic module the angle is fixed and, therefore, positioning of the stone is limited to the one direction only.

Successful ESWL treatment in obese patients (weight range $135-181 \mathrm{~kg}$ ) was reported with an overall stone free rate at 3 months of $68 \%$ (3) compared to $80-85 \%$ in non-obese patients (5). In the aforementioned study (3) higher energy settings were implemented to achieve successful stone fragmentation in morbidly obese patients but no serious complications were recorded.
In our study the overall stone free rate of $73 \%$ was achieved. Continuous ultrasound monitoring during lithotripsy gives the accurate information of fragmentation of all types of kidney stones. That together with accurate location of the stone into the nearest to the focal area point using the overtable module eliminate the importance of using higher energy setting. As a result we had no complication such as subcapsular haematoma or acute pyelonephritis. Furthermore, increasing the number of treatment sessions allows to perform "gentle" fragmentation of stones with stone fragments $1-2 \mathrm{~mm}$. The mean number of treatments per patient in our study was 2.1. As a consequence only $2(5.4 \%)$ of our patients required hospitalization for post-ESWL pain.

Using the overtable module increases security of the table. The table was lowered (the z-axis) to its limit and was covered with the protected plastic cover to ensure that the table was secure. The latter was technically impossible with the fluoroscopic module.

A higher radiation dose was previously reported with the markedly obese patients (43 versus $12 \mathrm{rad}$ ) due to radiographic technique (increased kilo voltage and milliampers per second) required to penetrate the larger body to obtain satisfactory radiographs (12). In our study we only applied ultrasound location, which eliminated the risk of radiation.

The lowest success rate was found in the patients treated with the blast path between 2 and $3 \mathrm{~cm}$. Nine out of 14 patients $(64.3 \%)$ were treated successfully with the aforementioned technique.

The management of stone disease is of great economic importance. In the US the cost to the taxpayer for the evaluation and management of urinary tract stone disease in 1993 was US\$ 1.23 billion and a further US\$ 139 million of wages were lost as a result of the disease (13). The most cost-efficient treatment in our study was in the patients with pelvic stones. The cost of treatment of the patients with pelvic stones was US\$ 349 compared to US\$ 633 for treatment of the patients with low caliceal stones.

The success rate of the treatment of the patients with low caliceal stones where the stone was positioned more than $1 \mathrm{~cm}$ from the focal point on the extended shock pathway was only $50 \%$. 


\section{CONCLUSION}

We conclude that ESWL with the Siemens Lithostar-plus is the most effective and cost-efficient in obese patients with pelvic stones sized between 6 and $20 \mathrm{~mm} .87 \%$ success rate was achieved The increased distance from the skin surface to the stone in those patients do not decrease the success rate provided the stone is positioned in the focal point or within $3 \mathrm{~cm}$ of it on the extended shock pathway. ESWL should not be considered as the first line of treatment in the patients with low caliceal stones where the stone was positioned more than $1 \mathrm{~cm}$ from the focal point on the extended shock pathway.

\section{REFERENCES}

1. Word Health Organization. Obesity. Preventing and Managing the Global Epidemic. Geneva. World Health Organization. 1997.

2. Koo BC, Burtt G, Burgess NA: Percutaneous stone surgery in the obese: outcome stratified according to body mass index. BJU Int. 2004; 93: 1296-9.

3. Thomas R, Cass AS: Extracorporeal shock wave lithotripsy in morbidly obese patients. J Urol. 1993; 150: 30-2.

4. Troy AJ, Anagnostou T, Tolley DA: Flexible upper tract endoscopy. BJU Int. 2004; 93: 671-9.
5. Peterson RF., Lifshitz DA, Kuo RL., Siqueira TM Jr, Lingeman JE: Shock wave lithotripsy monotherapy for renal calculi. Int Braz J Urol. 2002; 28: 291-301.

6. Choban PS, Flancbaum L: The impact of obesity on surgical outcomes: a review. J Am Coll Surg. 1997; 185: 593-603.

7. Kaminsky J, Gadaleta D: A study of discrimination within the medical community as viewed by obese patients. Obes Surg. 2002; 12: 14-8.

8. Carson CC 3rd, Danneberger JE, Weinerth JL: Percutaneous lithotripsy in morbid obesity. J Urol. 1988; 139: 243-5.

9. Whelan JP, Finlayson B, Welch J, Newman RC: The blast path: theoretical basis, experimental data and clinical application. J Urol. 1988; 140: 401-4.

10. Folberth W, Kohler G, Rohwedder A, Matura E: Pressure distribution and energy flow in the focal region of two different electromagnetic shock wave sources. J Stone Dis. 1992; 4: 1-4.

11. Loske AM, Prieto FE, Gutierrez J, Zendejas H, Saita A, Velez Gomez E: Evaluation of a bifocal reflector on a clinical lithotripter. J Endourol. 2004; 18: 7-15; discussion 15-6.

12. Cass AS: Equivalence of mobile and fixed lithotriptors for upper tract stones. J Urol. 1991; 146: 290-3.

13. May DJ, Chandhoke PS: Efficacy and cost-effectiveness of extracorporeal shock wave lithotripsy for solitary lower pole renal calculi. J Urol. 1998; 159: 247.

Received: October 18, 2004

Accepted after revision: December 5, 2004

\author{
Correspondence address: \\ Dr. V. A. Mezentsev \\ 19 Old Mill View Dewsbury \\ West Yorkshire, WF12 9QJ, UK \\ Phone: + 441924451342 \\ E-mail: vitalimezentsev@ hotmail.com
}

